\title{
ESPECIES RARAS DE HONGOS ANAMORFOS SAPROBIOS EN EL ESTADO DE TABASCO
}

\author{
Cinthya Ivonne Becerra-Hernández ${ }^{1,4}$, Gabriela Heredia ${ }^{1}$, Rosa María Arias ${ }^{1}$, \\ Rafael F. Castañeda-Ruiz ${ }^{2}$ y Julio Mena-Portales ${ }^{3}$ \\ ${ }^{1}$ Instituto de Ecología, A.C., km 2.5 antigua carretera a Coatepec Núm. 351, \\ Congregación el Haya, 91070 Xalapa, Veracruz, México \\ ${ }^{2}$ Instituto de Investigaciones Fundamentales en Agricultura \\ Tropical “Alejandro de Humboldt”, La Habana, Cuba. \\ ${ }^{3}$ Instituto de Ecología y Sistemática, Carretera de Varona km. 3.5, \\ Apdo. postal 8029, Capdevila, Boyeros, La Habana, Cuba. \\ ${ }^{4}$ Autor para la correspondencia: ivi170@gmail.com
}

\section{RESUMEN}

Se describen 15 especies de hongos anamorfos saprobios colectados en un fragmento de selva del Centro de Interpretación y Convivencia con la Naturaleza Yumka’ en el estado de Tabasco. En todos los casos el número de registros a nivel mundial son escasos, razón por la que los consideramos como raros. De las especies estudiadas, seis son nuevos reportes para el neotrópico y todas representan nuevos registros para México y Tabasco; ocho de ellas no habían sido recolectadas desde su descripción original. Se propone el nombre nuevo Sporidesmium multiforme nom. nov para Sporidesmium polymorphum R.F. Castañeda. Para cada especie se incluye su descripción morfológica, ilustraciones e información sobre su distribución mundial conocida hasta la fecha. Las descripciones fueron realizadas con material extraído directamente de los restos vegetales.

Palabras clave: anamorfos, diversidad, hongos conidiales, hongos tropicales, México.

\begin{abstract}
A total of 15 species of saprobe anamorphic fungi collected in a fragment of jungle of the Centro de Interpretación y Convivencia con la Naturaleza Yumka' in the state of Tabasco are described. For all species studied, the number of records worldwide are scarce, a reason
\end{abstract}


for which we consider them to be rare. Of the species studied, six are new reports for the Neotropical region and all represent new records for Mexico and Tabasco. In addition, eight of them had not been reported since their original description. A morphological description, illustrations and up-to-date known world distributions are included for each taxon. A new name Sporidesmium multiforme nom. nov is proposed for Sporidesmium polymorphum R.F. Castañeda. The descriptions that appear were done from material extracted directly from the leaf litter.

Key words: anamorphic fungi, conidial fungi, diversity, Mexico, tropical fungi.

\section{INTRODUCCIÓN}

Los hongos anamorfos o conidiales son aquellos a los que únicamente se les ha observado la reproducción mediante procesos asexuales (Herrera y Ulloa, 1990). Lo anterior puede deberse al desconocimiento de los factores que inducen sus mecanismos sexuales o bien que a lo largo de su evolución hayan perdido esta capacidad (Kendrick, 1992). Representan un grupo diverso y ampliamente distribuido en la naturaleza, pueden ser parásitos o saprobios, estos últimos colonizan todo tipo de restos vegetales en diferente estado de descomposición (Subramanian, 1983). Por su exuberancia y condiciones climatológicas, es de suponer que los ambientes tropicales resguardan una alta riqueza de anamorfos. En las regiones cálido-húmedas de México el conocimiento sobre las especies saprobias anamorfas es incipiente, por lo que es importante intensificar las exploraciones que registren la riqueza de tales organismos que habitan sobre los restos vegetales de estas áreas. Para el estado de Tabasco son muy pocos los estudios que incluyen especies anamorfas como parte de su diversidad fúngica (Heredia et al., 2006; Becerra-Hernández, et al., 2007; Heredia et al., 2008; Becerra-Hernández et al., 2008).

\section{MÉTODOS}

Se colectaron hojas, troncos y ramas en diferentes estados de descomposición en el área ocupada por selva mediana subperennifolia del Centro de Interpretación y Convivencia con la Naturaleza Yumka' (CICN Yumka'); una descripción detallada del área de estudio se encuentra en Becerra-Hernández et al., 2007. El material se trasladó en bolsas de papel al laboratorio, en donde fue colocado en cámaras húmedas. Durante un mes se revisó diariamente la humedad y aireación 
de las cámaras, posteriormente bajo el microscopio estereoscópico se extrajeron las estructuras de reproducción para la elaboración de preparaciones permanentes con alcohol polivinílico, y semi-permanentes con ácido láctico (Muller et al., 2004). Para la identificación se consultó la bibliografía especializada para cada caso. El material de referencia se encuentra depositado en la colección de hongos microscópicos del Laboratorio de Micromicetos del Instituto de Ecología, A.C., Xalapa, Veracruz (XAL).

\section{RESULTADOS}

Los 15 taxa que a continuación se describen han sido registrados en muy pocas ocasiones, ocho de ellos no lo habían sido desde su descripción original. Todas las especies representan nuevos registros para México y el estado de Tabasco. Chaetopsina romantica Rambelli \& Lunghini, Dictyochaeta plovercovensis Goh \& Hyde, Dictyochaetopsis menisporoides Aramb. \& Cabello, Helminthosporium zombaense Sutton, Hemibeltrania cinnamomi (Deighton) Piroz. y Porobeltraniella porosa (Piroz. \& S.D. Patil) Gusmão se describen por primera ocasión para la región Neotropical. Del material colectado, 13\% se considera como especies foliícolas, $40 \%$ lignícolas y $47 \%$ plurívoras, entendiéndose por foliícolas a aquellas que habitan en hojas, lignícolas las que viven en madera y plurívoras las que se nutren de muy diversas sustancias, que pueden encontrarse en los más diferentes sustratos (Ulloa y Hanlin, 2006). Cabe mencionar que el conocimiento de esta preferencia por el sustrato no es definitivo debido a la escasa información que se tiene de las especies presentadas. Se propone Sporidesmium multiforme como nombre nuevo para Sporidesmium polymorphum R.F. Castañeda (Castañeda-Ruiz, 1986), homónimo posterior de la especie descrita por Corda en 1837.

\section{DESCRIPCIÓNES MORFOLÓGICAS}

Anungitopsis speciosa R.F. Castañeda \& W.B. Kendr. 1990. University of Waterloo Biology Series 33: 9. (Figs. 1a-1c).

Colonias dispersas, anfígenas, pilosas, de color café. Micelio inmerso en el sustrato; hifas ramificadas, lisas, septadas, de color café. Conidióforos macronematosos, solitarios o en grupos, erectos, rectos o flexuosos, simples, cilíndricos, lisos, septados, base café, aclarándose hacia el ápice, 148-270 × 3-5 $\mu \mathrm{m}$. Células conidiógenas poli- 
blásticas, integradas, terminales, simpodiales, lisas, subhialinas. Conidios catenulados, secos, acrógenos, cilíndricos, con los extremos redondeados o puntiagudos y con una cicatriz estrecha, lisos, 2-5 septos, subhialinos o cafés pálidos, 16-21.5 × 3-5 $\mu \mathrm{m}$.

Material examinado: TABASCO, CICN Yumka', Villahermosa, Centro. Col. C. Becerra y S. Cornelio, 24/octubre/2006. CB1404. En tronco en descomposición.

Distribución y sustratos registrados: Especie previamente conocida sólo para Cuba en donde fue colectada sobre hojas caídas de Buchenavia capitata Eichler (Castañeda-Ruiz y Kendrick, 1990).

Arachnophora polyradiata (Mercado \& R.F. Castañeda) R.F. Castañeda \& W. Gams. 1997. Nova Hedwigia 64(3-4): 482. (Figs. 2a-2e).

= Cacahualia polyradiata Mercado \& R.F. Castañeda. 1984. Rev. Jard. Bot. Nac. 5: 94.

Colonias dispersas, pilosas, cafés oscuras a negruzcas. Micelio inmerso en el sustrato; hifas ramificadas, septadas, lisas, de color café oscuro. Conidióforos macronematosos, solitarios o agrupados, erectos, rectos o flexuosos, simples, cilíndricos, lisos, septados, cafés a cafés oscuros, pálidos hacia el ápice, 126-154 × 4.5-5 $\mu \mathrm{m}$; con proliferaciones percurrentes. Células conidiógenas monoblásticas, integradas, percurrentes, anilladas, lisas, cafés a cafés pálidas. Conidios solitarios, secos, acrógenos, estaurospóricos, conidio completo 30-40.5 × 25-35 $\mu \mathrm{m}$; 2 células centrales angulares, siendo mayor la que surge de la célula basal, septos muy oscuros y constreñidos, cafés oscuras, viéndose a menudo en diferentes planos; 18.5-22 × 12-15 $\mu \mathrm{m}$. Los brazos surgen radialmente, en su mayoría en número de 5-7, septados, la base forma una célula obcónica café, el brazo se va estrechando y termina en un ápice aguzado subhialino, 16.5-20 × 6-7 $\mu \mathrm{m}$, estrechándose a $1 \mu \mathrm{m}$; del ápice de los brazos surgen 2-8 conidios, falciformes, filiformes o cilíndricos, hialinos, 4-6.5 × 0.5-1 $\mu \mathrm{m}$.

Material examinado: TABASCO, CICN Yumka', Villahermosa, Centro. Col. G. Heredia, C. Becerra y J. Mena, 9/junio/2006. CB1136, CB1163. En rama leñosa y tronco en descomposición.

Distribución y sustratos registrados: A la fecha esta especie sólo se conocía para Cuba, el material tipo fue colectado en un fruto semipodrido de Tamarindus indica L. y sobre ramas muertas de Castanospermum australe A. Cunn \& Fraser (Mercado-Sierra y Castañeda-Ruiz, 1984), posteriormente se encontró en restos de Roystonea regia (H.B.K.) O.F. Cook (Delgado y Mena-Portales, 2004a).

Ceratosporium caribense Hol.-Jech. 1988. Česká Mykol. 42(1): 29. (Figs. 3a-3c). Sinanamorfo: Selenosporella (Figs. 3a-c). 
Colonias dispersas, pilosas, oliváceas oscuras a negras. Micelio en parte inmerso, en parte superficial; hifas ramificadas, septadas, lisas, cafés. Conidióforos micronematosos, dispersos, denticulados, cafés, 7-10 ×6.5-9 $\mu \mathrm{m}$. Células conidiógenas monoblásticas, integradas, intercalares, determinadas, cilíndricas, denticuladas. Conidios solitarios, secos, acrógenos, quiroides, compuestos de 5-6 brazos unidos y comprimidos en la base, los extremos distales de los brazos son rectos o ligeramente inclinados y divergentes, lisos, septados, cafés, más claros hacia el ápice, 185-335 × 7.5-16 $\mu \mathrm{m}$, adelgazándose hacia la punta, 5.5-8 $\mu \mathrm{m}$.

Sinanamorfo: Tipo Selenosporella, se puede originar en las hifas o en la zona distal de los brazos. Células conidiógenas poliblásticas, integradas, terminales, simpodiales, lageniformes, dispuestas generalmente en verticilos, subhialinas, 8-19.5 $\times$ 3-4 $\mu \mathrm{m}$. Conidios filiformes, aseptados, hialinos, pared delgada, 3-4.5 $\times 0.5 \mu \mathrm{m}$.

Material examinado: TABASCO, CICN Yumka', Villahermosa, Centro. Col. G. Heredia, C. Becerra y J. Mena, 9/junio/2006. CB1127. En rama leñosa en descomposición.

Distribución y sustratos registrados: Esta especie sólo se conocía para Cuba, fue colectada creciendo sobre una rama en descomposición (Holubová-Jechová, 1988).

Chaetopsina romantica Rambelli \& Lunghini. 1979. Trans. Br. Mycol. Soc. 72(3): 491. (Figs. 4a-4b).

Colonias dispersas, pilosas, brillosas, de color café a café oscuro. Micelio en parte superficial, mayormente inmerso; hifas ramificadas, septadas, lisas, subhialinas. Conidióforos setiformes, macronematosos, solitarios o en pares, erectos, rectos, simples o ramificados, ligeramente verrugosos, septados, amarillos, 139-219 × 4-6 $\mu \mathrm{m}$; base bulbosa, se adelgazan gradualmente hacia el ápice; aparato conidiógeno situado en la parte basal, media y ápice de la seta. Células conidiógenas monofialídicas, discretas, determinadas, con un prominente collarete, mayormente ampuliformes o en forma de reloj de arena, nacen terminal o lateralmente de las hifas o directamente de los conidióforos setiformes, 7-27 × 2.5-3 $\mu \mathrm{m}$. Conidios agrupados, húmedos, acrógenos, cilíndricos, rectos, obtusos en los extremos, lisos, sin septos, hialinos, 9-10 × 1.5-2 $\mu \mathrm{m}$; agregados en una masa mucilaginosa.

Material examinado: TABASCO, CICN Yumka', Villahermosa, Centro. Col. G. Heredia, C. Becerra y J. Mena, 9/junio/2006. CB1345, CB1346, CB1348, CB1366, CB1367. En tronco, hoja y rama leñosa en descomposición.

Distribución y sustratos registrados: Costa de Marfil, en corteza no identificada (Rambelli y Lughini, 1979) y en Alabama, Estados Unidos en hojas muertas de Magnolia virginiana L. (Morgan-Jones, 1982). 
Cryptophialoidea ramosa Delgado, J. Mena \& Gené. 2005. Fungal Diversity 20: 31. (Figs. 5a-5b).

Colonias dispersas, pilosas, cafés. Micelio en parte superficial, en parte inmerso; hifas ramificadas, lisas, septadas, cafés pálidas a cafés. Conidióforos setiformes macronematosos, solitarios, rectos, ápice flexuoso, lisos, septados, cafés, el ápice ramificado 1-3 veces en ramas dicotomizadas, $145-172 \times 5-6.5 \mu \mathrm{m}$, ramas subuladas, septadas, cafés con las puntas más claras. Zona conidiógena cilíndrica, comprende 1 sola fila de fiálides arregladas estrechamente en bultos discretos y abiertos en un solo lado del estípite, 3-6 fiálides por bulto, con un estrecho campo de células estériles alrededor de la base de los bultos, 35-44.5 $\mu \mathrm{m}$ de largo. Células conidiógenas monofialídicas, discretas, determinadas, lageniformes, cafés pálidas, 11-16 × 2.5-4 $\mu \mathrm{m}$; collarete infundibuliforme. Conidios agrupados, húmedos, acrógenos, falcados con el extremo basal redondeado, lisos, septo 1 , hialinos, $19-22 \times 1.5 \mu \mathrm{m}$, producidos en masas mucilaginosas.

Material examinado: TABASCO, CICN Yumka', Villahermosa, Centro. Col. C. Becerra y S. Cornelio, 24/octubre/2006. CB1315, CB1399. En hoja y tronco en descomposición.

Distribución y sustratos registrados: Especie colectada en Cuba sobre hojas muertas de Ocotea nemodaphne Mez, Calyptranthes caroli Griseb., Beilschmiedia pendula (Sw.) Hemsl y hojas muertas no identificadas (Delgado et al., 2005), también ha sido registrada de Brasil en hojas muertas no identificadas y de Anacardium occidentale L. (Marques et al., 2008).

Dactylaria monticola R.F. Castañeda \& W.B. Kend., 1991, Univ. Waterloo Biol. Ser. 35: 30. (Figs. 6a-6c).

Colonias dispersas, pilosas o cespitosas, anfígenas, blancas. Micelio en parte superficial, en parte inmerso; hifas ramificadas, lisas, septadas, hialinas. Conidióforos macronematosos, solitarios, simples u ocasionalmente ramificados, rectos o flexuosos, septados, lisos, base café aclarándose hacia el ápice, 30-33 × 1.5-2 $\mu \mathrm{m}$. Células conidiógenas poliblásticas, simpodiales, terminales, integradas o discretas, región apical ligeramente hinchada. Conidios solitarios, secos, acrógenos, fusiformes, lisos, septo 1, subhialinos, 24.5-30 × 1-1.5 $\mu \mathrm{m}$; base truncada y ápice redondeado.

Material examinado: TABASCO, CICN Yumka', Villahermosa, Centro. Col. C. Becerra y S. Cornelio, 24/agosto/2006. CB1603. En hoja en descomposición.

Distribución y sustratos registrados: A la fecha Dactylaria monticola sólo se conocía para Cuba, el material tipo fue colectado en hojas muertas de Andira inermis (W. Wright) Kunth ex DC. (Castañeda-Ruiz y Kendrick, 1991). 
Dictyochaeta minutissima A. Hern. Gut. \& J. Mena. 1996. Mycol. Res. 100(6): 687. (Figs. 7a-7c).

Colonias dispersas, pilosas, blancas grisáceas. Micelio inmerso en el sustrato; hifas ramificadas, lisas, septadas, subhialinas. Conidióforos macronematosos, solitarios o agrupados, erectos, rectos o flexuosos, simples o algunas veces con ramificaciones laterales, cilíndricos, lisos, septados, base café pálida aclarándose hacia el ápice, 46.5-68.5 × 3-3.5 $\mu \mathrm{m}$. Células conidiógenas monofialídicas, integradas, terminales, 1-3 proliferaciones percurrentes, lageniformes, con cuello pero sin collarete definido. Conidios aglutinados, húmedos, acrógenos, acerosos a fusiformes, redondeados en el ápice y agudos en la base, rectos a ligeramente curvos, lisos, sin septos, hialinos a subhialinos, 6-11 × 0.5-0.98 $\mu \mathrm{m}$; sétula ausente; agregados en una masa mucilaginosa.

Material examinado: TABASCO, CICN Yumka', Villahermosa, Centro. Col. G. Heredia, C. Becerra y J. Mena, 9/junio/2006. CB1561. En hoja de palma en descomposición.

Distribución y sustratos registrados: Esta especie sólo se conocía para Cuba, fue colectada en pecíolos de hojas muertas de Coccothrinax miraguama Becc. (Hernández-Gutiérrez y Mena-Portales, 1996).

Dictyochaeta plovercovensis Goh \& K.D. Hyde. 1999. Fungal Diversity 3: 69. (Figs. 8a-8c).

Colonias dispersas, pilosas, cafés a cafés oscuras. Micelio mayormente inmerso; hifas ramificadas, lisas, septadas, subhialinas. Conidióforos setiformes, erectos, rectos, cilíndricos, adelgazándose hacia el ápice, lisos, septados, cafés oscuros, aclarándose gradualmente hacia el ápice, 188.5-259 × 5-6 $\mu \mathrm{m}$. Conidióforos macronematosos, 3-5 en fascículos alrededor del conidióforo setiforme, erectos, flexuosos, simples, clavados, lisos, 3-5 septos, cafés amarillentos, aclarándose hacia el ápice, 63-78.5 × $3 \mu \mathrm{m}$; con un collarete infundibuliforme. Células conidiógenas mono o polifialídicas, integradas, terminales, lisas, cafés pálidas. Conidios agrupados, húmedos, acrógenos, ligeramente curvos, asimétricos, redondeados en el extremo distal, ligeramente agudos en el proximal, lisos, sin septos, hialinos, 12-15 × 2-2.5 $\mu \mathrm{m}$; con una sétula filiforme, simple en cada extremo, 5-10 $\mu \mathrm{m}$; agregados en masas mucilaginosas hialinas.

Material examinado: TABASCO, CICN Yumka', Villahermosa, Centro. Col. G. Heredia, C. Becerra y J. Mena, 9/junio/2006. CB1557. En hoja en descomposición.

Distribución y sustratos registrados: Este taxon no había sido citado desde que fue descrito de Hong Kong creciendo sobre madera sumergida en descomposición (Goh y Hyde, 1999). 
Acta Botanica Mexicana 96: 15-31 (2011)
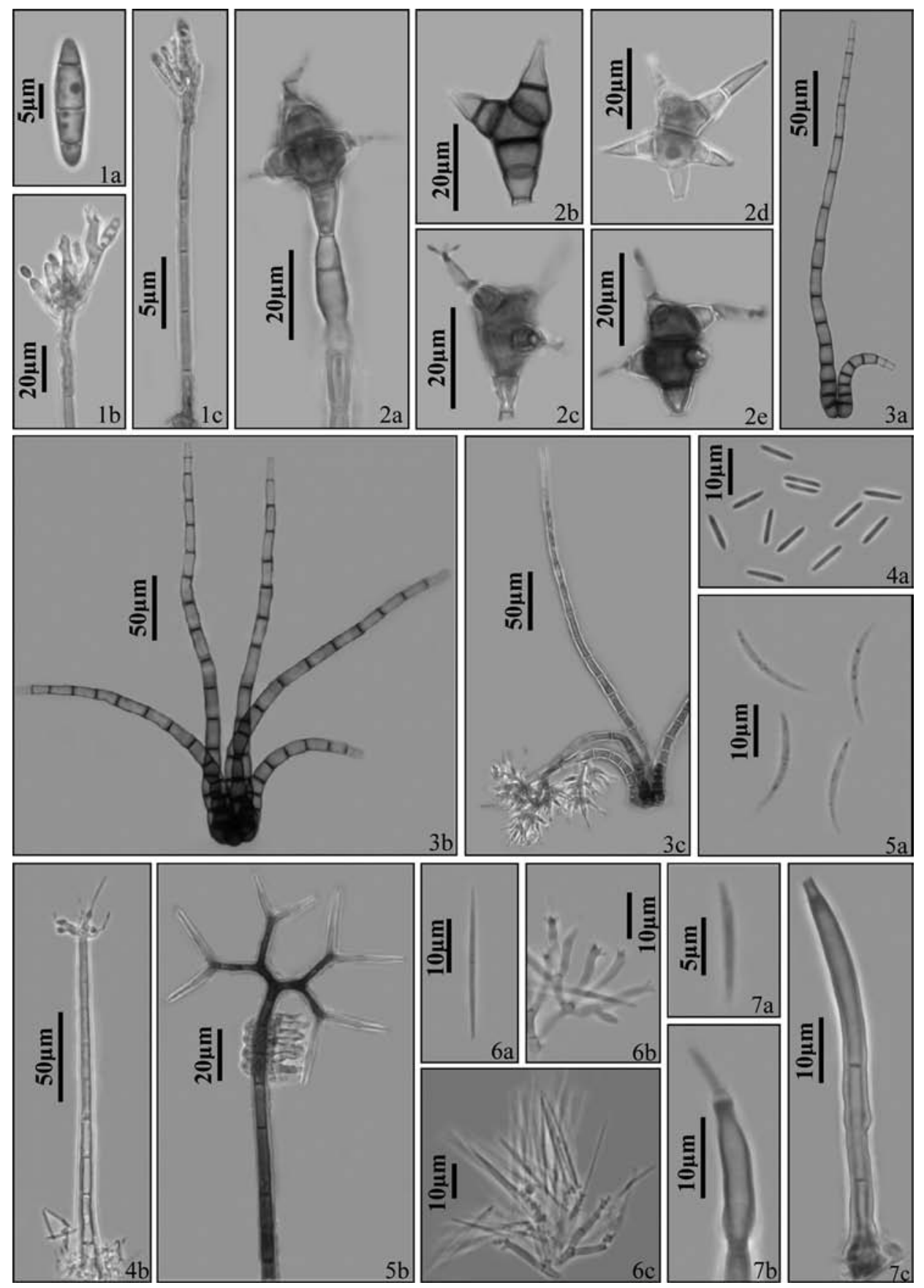

Pie de figura en la página 21. 
Dictyochaetopsis menisporoides (Hol.-Jech.) Aramb. \& Cabello. 1990. Mycotaxon 38: 13. (Figs. 9a-9c).

= Dictyochaeta menisporoides Hol.-Jech. 1984. Folia Geobot. Phytotax. 19(4): 430.

Colonias dispersas, pilosas, café grisáceas a gris blancuzcas cuando están esporulando. Micelio mayormente inmerso; hifas ramificadas, lisas, septadas, cafés pálidas. Conidióforos de dos tipos: a) setiformes, solitarios, dispersos, erectos, rectos, cilíndricos, lisos, septados, ápice fértil, 140-197 × 4-5 $\mu \mathrm{m}$, con fiálides distribuidas de la base a la punta, rectos o ligeramente curvos, b) simples, solitarios o agrupados alrededor del conidióforo setiforme, erectos, rectos, cilíndricos, lisos, base café pálida, ápice subhialino, 20-25 × 3-3.5 $\mu \mathrm{m}$, con un collarete terminal y en ocasiones 1-2 collaretes laterales. Células conidiógenas polifialídicas, integradas o discretas, terminales o laterales, subhialinas a cafés pálidas, $12-21 \times 3-4 \mu \mathrm{m}$; collaretes infundibuliformes. Conidios agrupados, húmedos, acrógenos, fusiformes a cilíndricos, curvos, con los extremos puntiagudos, lisos, sin septos, hialinos, 13.5-16.5 $\times 2-2.5 \mu \mathrm{m}$; con una sétula filiforme, recta o ligeramente curva en cada extremo 7-10 $\mu \mathrm{m}$; agregados en masas mucilaginosas.

Material examinado: TABASCO, CICN Yumka', Villahermosa, Centro. Col. C. Becerra y S. Cornelio, 24/octubre/2006. CB1319; CB1322; CB1340; CB1445; CB1564. En hojas en descomposición.

Distribución y sustratos registrados: El ejemplar tipo fue colectado en Eslovaquia en frutos caídos de Quercus robur L. (Holubová-Jechová, 1984). El presente representa el primer registro para la región Neotropical.

Tomando en cuenta que en este hongo las células conidiógenas surgen de pequeñas ramificaciones a lo largo del conidióforo, a diferencia del resto de las especies del género Dictyochaeta, Arambarri y Cabello (1990) transfirieron la especie a Dictyochaetopsis.

Figs. 1-7. 1. Anungitopsis speciosa. a. conidio fusiforme., b. ápice del conidióforo con conidios simples y catenulados adheridos., c. conidióforo con células conidiógenas y conidios. 2. Arachnophora polyradiata. a. conidióforo con conidio pegado., b-e. diferentes estados de desarrollo del conidio. 3. Ceratosporium caribense. a. conidio con 2 brazos., b. conidio con 5 brazos., c. conidio con el sinanamorfo Selenosporella en dos de sus brazos. 4. Chaetopsina romantica. a. conidios cilíndricos., b. conidióforo setiforme con células conidiógenas y conidios adheridos. 5. Cryptophialoidea ramosa. a. conidios falcados., b. conidióforo con la zona conidiógena, fiálides abiertas hacia un lado del estípite. 6. Dactylaria monticola. a. conidio fusiforme., b. conidióforo ramificado con células conidiógenas denticuladas en el ápice., c. conidióforo ramificado con conidios adheridos. 7. Dictyochaeta minutissima. a. conidio aceroso., b. conidio saliendo de la célula conidiógena., c. conidióforo cilíndrico. 
Helminthosporium zombaense B. Sutton. 1993. Mycol. Pap. 167: 32. (Figs. 10a y 10b).

Colonias dispersas, pilosas, cafés oscuras. Micelio inmerso en el sustrato; hifas ramificadas, lisas, septadas, cafés pálidas. Conidióforos macronematosos, solitarios o en grupos de 2-3, erectos, rectos o ligeramente flexuosos, cilíndricos, lisos, septados, cafés oscuros, aclarándose hacia al ápice, 213-257 × $7 \mu \mathrm{m}$. Células conidiógenas politréticas, integradas, terminales y laterales, cilíndricas, lisas, cafés pálidas. Conidios solitarios o catenulados, 2 conidios por cadena, secos, acrógenos, rectos o ligeramente flexuosos, obclavados, curvos, lisos, 3-10 distoseptos, cafés pálidos a cafés oscuros, 21-50 × 7-10 $\mu \mathrm{m}$; cicatriz basal café oscura.

Material examinado: TABASCO, CICN Yumka', Villahermosa, Centro. Col. C. Becerra y S. Cornelio, 24/octubre/2006. CB1491. En raquis de corozo (Scheelea liebmannii Becc.).

Distribución y sustratos registrados: Este hongo no había sido colectado desde su descripción, la presente colecta es el primer registro de la especie para el Neotrópico. El material tipo proviene de Malawi, en donde fue encontrado sobre tallos de una liana no identificada (Sutton, 1993).

Hemibeltrania cinnamomi (Deighton) Piroz. 1963. Mycol. Pap. 90: 32. (Figs. 11a y 11b).

= Hansfordia cinnamomi Deighton. 1960. Mycol. Pap. 78: 14 .

Colonias dispersas, anfígenas, aterciopeladas, café grisáceas. Micelio en parte superficial, en parte inmerso en el sustrato; hifas ramificadas, lisas, subhialinas. Conidióforos macronematosos, solitarios o en grupos (2-3), surgen de la base de células radialmente lobuladas, simples o ramificados, erectos, rectos o flexuosos, cilíndricos, lisos, en la base café oliváceos, ápice subhialino, 185-236.5 × 4-5 $\mu \mathrm{m}$. Células conidiógenas poliblásticas, integradas, terminales e intercaladas, denticuladas, lisas; dentículos cónicos a cilíndricos, subhialinas. Conidios solitarios, secos, acrógenos, elipsoidales a obovoides, 1 dentículo en el extremo proximal y el extremo distal redondeado, lisos, subhialinos, 12-15.5 × 5-7 $\mu \mathrm{m}$.

Material examinado: TABASCO, CICN Yumka', Villahermosa, Centro. Col. C. Becerra y S. Cornelio, 24/octubre/2006. CB1464; CB1465. En hoja en descomposición.

Distribución y sustratos registrados: Esta especie fue descrita por Deighton quien tentativamente la nombró como Hansfordia cinnamomi (Deighton, 1960); el material tipo se encontró ocasionando manchas foliares en hojas vivas de Cinnamomum zeylanicum Breyne en Sierra Leona. Por el tipo de conidióforo que emerge 
de una célula radialmente lobulada y la forma rómbica de las esporas, Pirozynski reubicó a este taxon en el género Hemibeltrania (Pirozynski, 1963). También ha sido colectada en la India sobre hojas muertas de Cinnamomum sp. (Rao y De Hoog, 1986)

Phialogeniculata multiseptata Matsush. 1993. Matsush. Mycol. Mem. 7: 60. (Figs. 12a-12c).

Colonias dispersas, pilosas, oliváceas. Micelio mayormente inmerso; hifas ramificadas, lisas, septadas, subhialinas. Conidióforos macronematosos, solitarios, erectos, rectos, simples, cilíndricos, lisos, septados, cafés, aclarándose hacía el ápice, 51.5-68 × 2.5-3 $\mu \mathrm{m}$. Células conidiógenas monofialídicas, integradas, terminales, proliferaciones enteroblásticas percurrentes, collarines infundibuliformes. Conidios solitarios, secos, acrógenos, obclavados, lisos, 2-5 septos, hialinos, 19-27 × 3-4.5 $\mu \mathrm{m}$; gutulados.

Material examinado: TABASCO, CICN Yumka', Villahermosa, Centro. Col. G. Heredia, C. Becerra y J. Mena, 9/junio/2006. CB1274. En raquis de palma en descomposición.

Distribución y sustratos registrados: Esta especie sólo se conocía para el Perú, fue colectada sobre un pecíolo muerto de palma no identificada (Matsushima, 1993).

Phialogeniculata fue erecto por Matsushima en 1971, con la especie tipo $P$. guadacanalensis; entre las características que el autor menciona como distintivas del género está la forma obclavada de las esporas así como la ausencia de sétulas. Kuthubutheen y Nawawi (1991a) propusieron la invalidación del género y su reubicación dentro de Dictyochaeta Speg.; sin embargo dicha propuesta no ha sido aceptada por subsecuentes autores que han descrito nuevas especies dentro de Phialogeniculata (Matsushima, 1993; Hyde et al., 1998). La descripción genérica de Dictyochaeta señala que los conidios son hialinos, lisos, típicamente falcados pero pueden ser elipsoidales, clavados, fusiformes o cilíndricos, caracteres distintos al que presentan las especies de Phialogeniculata en el que los conidios son generalmente obclavados. Por tal razón consideramos conveniente ubicar el material estudiado dentro del género mencionado.

Porobeltraniella porosa (Piroz. \& S.D. Patil) Gusmão. 2004. Mycologia 96(1): 151. (Figs. 13a-13b).

= Beltraniella porosa Piroz. \& S.D. Patil. 1970. Can. J. Bot. 48(3): 573.

Colonias dispersas, anfígenas, aterciopeladas, gris negruzcas. Micelio en parte superficial, en parte inmerso; hifas ramificadas, lisas, septadas, subhialinas. 
Acta Botanica Mexicana 96: 15-31 (2011)
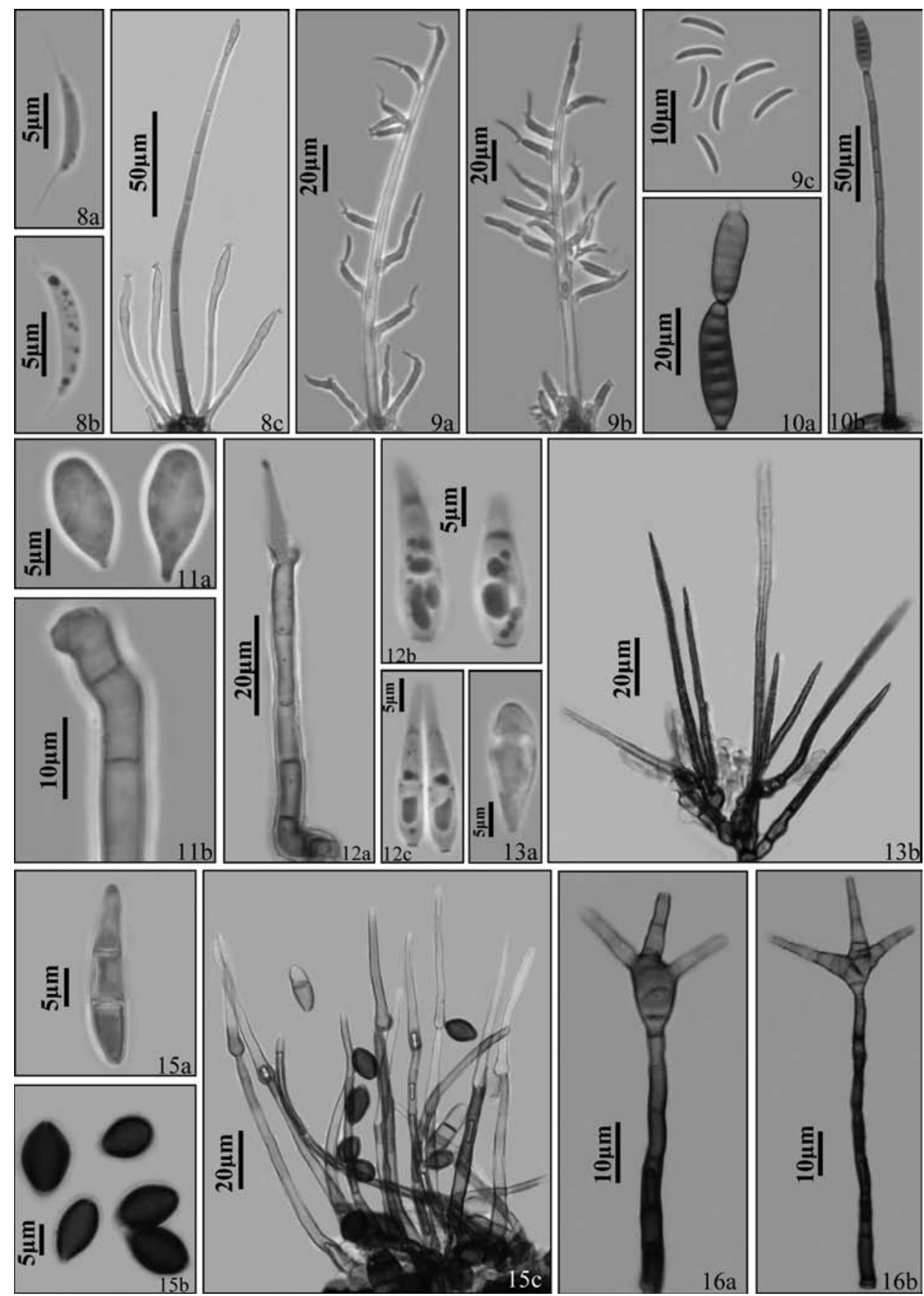

Pie de figura en la página 25. 
Conidióforos setiformes, macronematosos, solitarios o en grupos pequeños, erectos, ramificados, lisos, septados, café oliváceos pálidos, ligeramente más oscuros de la base, 81.2-148 × 4-5 $\mu \mathrm{m}$; nacen en la base de células radialmente lobuladas. Células conidiógenas poliblásticas, integradas, terminales, elipsoidales a obovoides, denticuladas, 1-3 dentículos, lisas, subhialinas. Células separadoras terminales, integradas, elipsoidales, subhialinas, 1 dentículo en cada extremo. 10-12 × $4 \mu \mathrm{m}$. Conidios solitarios, secos, acrógenos, turbinados, extremo proximal rostrado, extremo distal truncado, lisos o finamente verrugosos, sin septos, subhialinos, 17-20 $\times 5-7 \mu \mathrm{m}$; con unos poros circulares cerca de la parte más ancha.

Material examinado: TABASCO, CICN Yumka', Villahermosa, Centro. Col. C. Becerra, 13/marzo/2005. CB1374. En rama leñosa en descomposición.

Distribución y sustratos registrados: El material tipo fue colectado en hojas muertas de Diospyros embryopteris Pers. y Gnetum ula Brongn en la India (Pirozynski y Patil, 1970), posteriormente fue reportado para Australia en hojas muertas no identificadas (Sutton, 1980). Inicialmente esta especie fue descrita como Beltraniella porosa Piroz. \& S.D. Patil (Pirozynski y Patil, 1970), en 2004 Gusmão la transfirió a un nuevo género, Porobeltraniella, debido a que los conidios presentan poros en vez de la característica banda transversal de las especies del género Beltraniella.

Sporidesmium multiforme R.F. Castañeda nom. nov.

Basónimo: Sporidesmium polymorphum R.F. Castañeda. 1986. Deuteromycotina de Cuba, Hyphomycetes IV (La Habana): 11. (Figs. 15a-15c).

Colonias dispersas, pilosas, anfígenas, cafés. Micelio parcialmente superficial y en parte inmerso; hifas ramificadas, lisas, septadas, cafés pálidas. Conidióforos

Figs. 8-16. 8. Dictyochaeta plovercovensis. a-b. conidios fusiformes con una sétula filiforme en cada extremo., c. conidióforo setiforme con conidióforos más pequeños alrededor de la base. 9. Dictyochaetopsis menisporoides. a-b. conidióforos setiformes con células conidiógenas discretas polifialídicas dispuestas a lo largo del estípite y conidios., c. conidios fusiformes con una sétula filiforme en cada extremo. 10. Helminthosporium zombaense. a. conidio catenulado., b. conidióforo con conidio adherido. 11. Hemibeltrania cinnamomi. a. conidios obovoides., b. ápice del conidióforo con pequeños dentículos. 12. Dictyochaeta multiseptata. a. conidióforo con conidio saliendo de la célula conidiógena., b-c. conidios obclavados. 12. Porobeltraniella porosa. a. conidio con poros circulares en la parte más ancha., b. conidióforo setiforme central y setas. 13. Sporidesmium multiforme. a. conidio obclavado., b. conidios elípticos o subesféricos., c. conidióforos con los dos tipos de conidios, algunos adheridos y otros desprendidos. 15. Triposporium lambdaseptatum. a-b. conidióforo con conidio adherido. 
macronematosos, solitarios o agregados, erectos, rectos o flexuosos, simples, en ocasiones ramificados, lisos, septados, cafés o fuscos, 80-125 × 5-7.5 $\mu \mathrm{m}$; con proliferaciones ampuliformes, alargadas. Células conidiógenas monoblásticas, integradas, terminales, cilíndricas o ampuliformes, percurrentes, cafés o cafés pálidas, 10$27.5 \times 2-3 \mu \mathrm{m}$. Conidios solitarios, secos, acrógenos, biformes: a) obclavados, lisos, 1-4 septos, subhialinos o cafés pálidos, 10-28 × 5-5.5 $\mu \mathrm{m}$. b) elípticos a subesféricos, lisos, sin septos, negros o cafés, 7-13 × 5-6 $\mu \mathrm{m}$; con un poro en el ápice.

Material examinado: TABASCO, CICN Yumka', Villahermosa, Centro. Col. C. Becerra, 13/marzo/2005. CB1112. En hoja en descomposición.

Distribución y sustratos registrados: Sporidesmium multiforme ha sido reportado en dos ocasiones en Cuba, la primera (ejemplar tipo) en hojas caídas de Coccoloba uvifera L. (Castañeda-Ruiz, 1986) y la segunda sobre ramas sumergidas de Syzygium jambos (L.) Alston. (Delgado y Mena-Portales, 2004b); además se ha colectado en Venezuela en hojas descompuestas caídas no identificadas (CastañedaRuiz et al., 2003).

Se propone el nombre nuevo para sustituir el homónimo posterior de Sporidesmium polymorphum publicado por Corda en 1837 (Corda, 1837), que de modo inadvertido fue publicado por R. Castañeda-Ruiz en 1986 en la serie Deuteromycotina de Cuba (Castañeda-Ruiz, 1986).

Triposporium lambdaseptatum (Matsush.) Kuthub. \& Nawawi. 1991. Mycol. Res. 95(2): 161. (Figs. 16a-16b).

= Ceratosporella lambdaseptata Matsush. 1971. Microfungi of the Solomon Islands and Papua-New Guinea: p. 11.

= Triposporium novoguineense M. Rifai. 1972. Reinwardtia 8: 336.

Colonias dispersas, inconspicuas, cafés pálidas a cafés. Micelio en parte superficial, en parte inmerso, hifas ramificadas, lisas, septadas, subhialinas. Conidióforos macronematosos, solitarios o en fascículos, erectos, flexuosos, curvos, simples, cilíndricos, lisos, septados, cafés oscuros, aclarándose hacia el ápice, 45-82 × 4-5 $\mu \mathrm{m}$; hasta 4 sucesivas proliferaciones percurrentes. Células conidiógenas monoblásticas, integradas, terminales, determinadas o percurrentes, lisas, cafés pálidas. Conidios solitarios, secos, acrógenos, estauriformes, célula basal truncada y café pálida, lisos, septados, cafés pálidos, 25-41 × 3.5-5.5 $\mu \mathrm{m}$; brazos 24.5-37 × 3.5-7 $\mu \mathrm{m}$ ancho base y 2.5-3 $\mu$ m ancho ápice.

Material examinado: TABASCO, CICN Yumka', Villahermosa, Centro. Col. G. Heredia, C. Becerra y J. Mena, 9/junio/2006. CB1298. En rama leñosa en descomposición. 
Distribución y sustratos registrados: Este hongo ha sido colectado en troncos y ramas muertas de Nueva Guinea (Matsushima, 1971; Rifai, 1972). También existen registros para Malasia (Kuthubutheen y Nawawi, 1991b) y para Perú (Matsushima, 1993).

\section{AGRADECIMIENTOS}

Los autores agradecen al Instituto de Ecología, A.C. Xalapa, a la Comisión Nacional para el Conocimiento y Uso de la Biodiversidad (Proyecto CONABIOEE003) y al Programa CYTED a través de la red XII.J (REDEMIC), por el apoyo económico brindado para la realización de este trabajo. Así como también a la M.V.Z. Laura E. Palacios del Centro de Interpretación y Convivencia con la Naturaleza (CICN) Yumka' las facilidades otorgadas para la colecta del material de estudio; a la Dra. Silvia Cappello García y al Biol. Sergio Alberto Gómez Cornelio de la Universidad Juárez Autónoma de Tabasco el apoyo otorgado en la misma fase de este trabajo.

\section{LITERATURA CITADA}

Arambarri, A. y M. Cabello. 1990. Considerations about Dictyochaeta, Codinaeopsis and a new genus: Dictyochaetopsis. Mycotaxon 38: 11-14.

Becerra-Hernández, C. I., G. Heredia y R. M. Arias. 2007. Contribución al conocimiento de los hongos anamorfos saprobios del Estado de Tabasco. II. Rev. Mex. Micol. 24: 39-53.

Becerra-Hernández, C. I., G. Heredia, R. M. Arias, J. Mena-Portales y R. F. Castañeda-Ruiz. 2008. Contribución al conocimiento de los hongos anamorfos saprobios del Estado de Tabasco. III. Rev. Mex. Micol. 28: 25-39.

Castañeda-Ruiz, R. F. 1986. Deuteromycotina de Cuba. Hyphomycetes IV. Instituto de Investigaciones Fundamentales en Agricultura Tropical "Alejandro Humboldt”. La Habana. 17 pp.

Castañeda-Ruiz, R. F. y B. Kendrick. 1990. Conidial fungi from Cuba: II. Univ. Waterloo Biol. Ser. 33: 1-61.

Castañeda-Ruiz, R. F. y B. Kendrick. 1991. Ninety-nine conidial fungi from Cuba and three from Canada. Univ. Waterloo Biol. Ser. 35: 1-132.

Castañeda-Ruiz, R. F., T. Iturriaga, D. W. Minter, M. Saikawa, G. Vidal y S. VelásquezNoa. 2003. Microfungi from Venezuela. A new species of Brachydesmiella, a new combination, and new records. Mycotaxon 85: 211-229. 
Corda, A. C. J. 1837. Icones fungorum hucusque cognitorum 1: 1-32.

Deighton, F. C. 1960. African fungi. I. Mycol. Pap. 78: 1-43.

Delgado-Rodríguez, G. y J. Mena-Portales. 2004a. Hifomicetos (hongos anamórficos) de la Reserva Ecológica “Alturas de Banao” (Cuba). Bol. Soc. Micol. Madrid 28: 115124.

Delgado-Rodríguez, G. y J. Mena-Portales, 2004b. Hifomicetos aero-acuáticos e ingoldianos de la Reserva de la Biosfera Sierra del Rosario (Cuba). Bol. Soc. Micol. Madrid 28: 105-113.

Delgado-Rodríguez, G., J. Mena-Portales, J. Gené y J. Guarro. 2005. New species of Cryptophialoidea and Hughesinia (hyphomycetes, anamorphic fungi) from Cuba. Fungal Divers. 20: 31-38.

Goh, T. K. y K. D. Hyde. 1999. Fungi on submerged wood and bamboo in the Plover Cove Reservoir, Hong Kong. Fungal Divers. 3: 57-85.

Gusmao, L. F. P. 2004. Porobeltraniella gen. nov. to accommodate two species of Beltraniella. Mycologia 96(1): 150-153.

Heredia, G., R. F. Castañeda-Ruiz, C. I. Becerra-Hernández y R. M. Arias. 2006. Contribución al conocimiento de los hongos anamorfos saprobios del Estado de Tabasco. I. Rev. Mex. Micol. 23: 53-62.

Heredia, G., R. M. Arias y C. I. Becerra-Hernández. 2008. Análisis del conocimiento de los hongos anamorfos saprobios en México. In: Heredia, G. (ed.). Tópicos sobre diversidad, ecología y usos de los hongos microscópicos en Iberoamérica. Programa Iberoamericano de Ciencia y Tecnología para el desarrollo (CYTED) e Instituto de Ecología, A.C., Xalapa, Ver. México. pp. 81-101.

Hernández-Gutiérrez, A. y J. Mena-Portales. 1996. Dictyochaeta minutissima sp. nov. on Coccothrinax miraguama from Cuba. Mycol. Res. 100(6): 687-688.

Herrera, T. y M. Ulloa. 1990. El reino de los hongos. Universidad Nacional Autónoma de México. México. 554 pp.

Holubová-Jechová, V. 1984. Lignicolous Hyphomycetes from Czekoslovakia 7. Chalara, Exochalara, Fusichalara and Dictyochaeta. Folia Geobot. Phytotax. 19: 387-438.

Holubová-Jechová, V. 1988. Studies on Hyphomycetes from Cuba VII. Seven new taxa of dematiaceous Hyphomycetes. Ceská Mykol. 42(1): 23-30.

Hyde, K. D., T. K. Goh y T. D. Steinke. 1998. Fungi on submerged wood in the Palmiet River, Durban, South Africa. S. Afr. J. Bot. 64(3): 151-162.

Kendrick, W. B. 1992. The fifth kingdom. 2a. ed. Focus Information Group Inc. Newburyport, MA. 406 pp.

Kuthubutheen, A. J. y A. Nawawi. 1991a. Dictyochaeta guadalcanalensis comb. nov. and several new records of the genus in Malaysia. Mycol. Res. 95(10): 1220-1223.

Kuthubutheen, A. J. y A. Nawawi. 1991b. A new species of Ceratosporella and Triposporium lambdaseptatum (Matsush.) comb. nov. from Malaysia. Mycol. Res. 95(2): 158-162.

Marques, M. F. O., A. C. R. Cruz, F. F. Barbosa y L. F. P. Gusmao. 2008. Cryptophiale and Cryptophialoidea (conidial fungi) from Brazil and keys to the genera. Rev. Bras. Bot. 31(2): 339-344.

Matsushima, T. 1971. Microfungi of the Solomon Islands and Papua-New Guinea. Published by the author. Kobe. 78 pp. 
Matsushima, T. 1993. Matsushima Mycological Memoirs No. 7. Matsushima Fungus Collect. Kobe. 75 pp.

Mercado-Sierra, A. y R. F. Castañeda. 1984. Cacahualia polyradiata, un hifomicete nuevo con conidios estaurospóricos. Rev. Jard. Bot. Nac. Univ. Habana 5: 89-101.

Morgan-Jones, G. 1982. Notes on Hyphomycetes. XLIII. Concerning Chaetopsina romantica. Mycotaxon 16: 192-196.

Muller, G. M., G. F. Bills y M. S. Foster. 2004. Biodiversity of fungi. Inventoring methods. Elsevier Academic Press. New York. 777 pp.

Pirozynski, K. A. 1963. Beltrania and related genera. Mycol. Pap. 90: 1-37.

Pirozynski, K. A. y S. D. Patil. 1970. Some setose Hyphomycetes of leaf litter in south India. Can. J. Bot. 48: 567-581.

Rambelli, A. y D. Lunghini. 1979. Chaetopsina species from tropical forest litter. Trans. Br. Mycol. Soc. 72(3): 491-494.

Rao, V. y G. S. De Hoog. 1986. New or critical Hyphomycetes from India. Stud. Mycol. 28: 1-84.

Rifai, M. A. 1972. A new tropical species of Triposporium. Reinwardtia 8: 365-367.

Subramanian, C. V. 1983. Hyphomycetes taxonomy and biology. Academic Press. Nueva York. 461 pp.

Sutton, B. C. 1980. Microfungi from Australian leaf litter. Proc. R. Soc. Qd. 91: 11-19.

Sutton, B. C. 1993. Mitosporic fungi from Malawi. Mycol. Pap. 167: 1-94.

Ulloa, M. y R. T. Hanlin. 2006. Nuevo diccionario ilustrado de micología. APS Press. St. Paul, Minnesota. 672 pp. 